\title{
Adaptation to biological motion leads to a motion and a form aftereffect
}

\author{
Stefanie Theusner • Marc H. E. de Lussanet • \\ Markus Lappe
}

Published online: 20 May 2011

(C) Psychonomic Society, Inc. 2011

\begin{abstract}
Recent models have proposed a two-stage process of biological motion recognition. First, template or snapshot neurons estimate the body form. Then, motion is estimated from body form change. This predicts separate aftereffects for body form and body motion. We tested this prediction. Observers viewing leftward- or rightward-facing point-light walkers that walked forward or backward subsequently experienced oppositely directed aftereffects in stimuli ambiguous in the facing or the walking direction. These aftereffects did not originate from adaptation to the motion of the individual light points, because they occurred for limitedlifetime stimuli that restrict local motion. They also occurred when the adaptor displayed a random sequence of body postures that did not induce the walking motion percept. We thus conclude that biological motion gives rise to separate form and motion aftereffects and that body form representations are involved in biological motion perception.
\end{abstract}

Keywords Biological motion - Adaptation and aftereffects $\cdot$ Neural mechanisms

\section{Introduction}

For many types of visual stimuli, prolonged viewing leads to adaptation aftereffects. For example, prolonged viewing

\author{
S. Theusner $(\bowtie) \cdot$ M. H. E. de Lussanet $\cdot$ M. Lappe \\ Institute of Psychology and Otto Creutzfeldt Center for Cognitive \\ and Behavioral Neuroscience, University of Muenster, \\ Fliednerstrasse 21, \\ 48149 Münster, Germany \\ e-mail: stefanie.theusner@uni-muenster.de \\ M. H. E. de Lussanet \\ e-mail: lussanet@uni-muenster.de \\ M. Lappe \\ e-mail:mlappe@uni-muenster.de
}

of a red surface leads to an apparent green tint of a subsequently viewed white surface, and prolonged viewing of continuous motion, such as a waterfall, leads to illusory opposite motion of a subsequently viewed static scene. An aftereffect provides evidence that a particular stimulus has a specific neuronal representation, because the aftereffect is based on the adaptation of neurons at a processing level that represents the stimulus (Clifford et al., 2007; Mather, Pavan, Campana, \& Casco, 2008). For example, the face aftereffect is based on the adaptation of face-selective neurons in face-processing areas of the cortex (e.g., Kovacs, Cziraki, Vidnyanszky, Schweinberger, \& Greenlee, 2008; Leopold, O’Toole, Vetter, \& Blanz, 2001; Webster, Kaping, Mizokami, \& Duhamel, 2004). Using this relation between the neural selectivity for a stimulus feature and the associated aftereffect, many studies on the motion aftereffect have traced the stages of motion processing in the cortex and have provided evidence of the existence of multiple motion representations: low-level stages processing local motion energy and high-level stages processing complex patterns of motion (for a review, see Mather et al., 2008).

Perception of biological motion is also a multistage process. Recent research has suggested that biological motion perception involves pathways in which, first, the form of the body is extracted from the stimulus and, thereafter, the motion of the body is determined from the responses of the body form detectors (Beintema \& Lappe, 2002; Giese \& Poggio, 2003; Lange \& Lappe, 2006). The models of Giese and Poggio and of Lange and colleagues (Lange, Georg, \& Lappe, 2006; Lange \& Lappe, 2006) contain such perceptual mechanisms. For example, the first stage in the model of Lange and Lappe (2006) consists of template cells that match the stimulus to templates of particular body postures (Fig. 1). Each template cell responds to a particular (preferred) configuration of the limbs. It becomes active when that particular configuration 


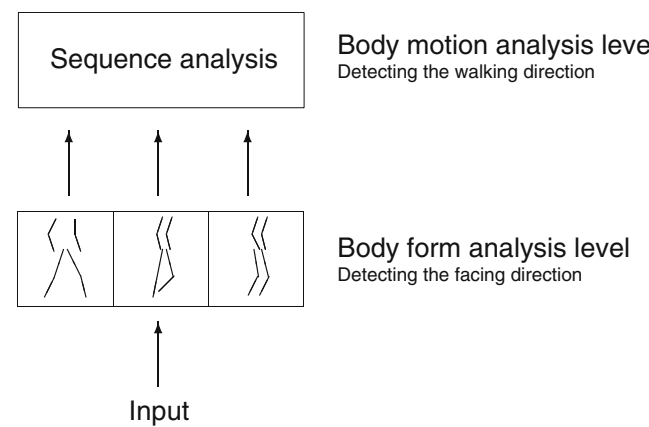

Fig. 1 Two stages in the perception of biological motion. At the body form analysis stage, the form of the stimulus is compared with the form of the human body by static template cells. At the body motion analysis stage, the temporal order in which those template cells are activated is analyzed

or posture is shown. Hence, over the course of the stimulus movement, different template cells are activated. The second stage, the body motion analysis stage, determines the temporal order of the activated template cells and, thus, the body motion.

The two stages have been used to predict human performance in different discrimination tasks of biological motion perception (Lange et al., 2006; Lange \& Lappe, 2006, 2007). In the first stage, form-dependent features of the stimulus - for example, the direction in which the figure is facing - can be extracted. To determine the facing direction, the maximal activity of all template cells belonging to a certain facing direction is fed into a temporal integrator neuron for that facing direction. This neuron is in competitive interaction with other facing direction neurons. The highest active integrator at decision time decides the perceived facing direction.

In the second stage, decisions about the motion direction of the stimulus-for example, forward or backward walking - can be drawn. Integrator neurons at this stage are sensitive to the temporal order in which the template cells are activated. Again, the most active integrator neuron determines the percept of forward or backward walking.

Because the model is separated into a body form analysis level and a body motion analysis level, it predicts that two different adaptation aftereffects can be observed for biological motion stimuli. At the body form analysis level, adaptation to the facing direction, or facing adaptation, should lead to the perception of the opposite facing direction. At the body motion analysis level, adaptation to the walking direction, or walking adaptation, should lead to the perception of the opposite walking direction.

In six experiments, we investigated the existence of the facing- and the walking-adaptation aftereffects and their dependence on form and motion features in the point-light stimulus.

\section{Method}

\section{Stimuli}

Point-light walker stimuli were derived from motiontracking data of a walking human seen from the side. Motion-tracking data were used for every stimulus. Translational motion of the hips was subtracted so that the walker walked in place as if on a treadmill. One walking cycle took $1.3 \mathrm{~s}$. The point-light stimuli consisted of white points on a black background. In most experiments, the light points were randomly distributed on the limb segments of the walker, following Beintema and Lappe (2002) and Beintema, Georg, and Lappe (2006). The points were redistributed anew for every new animation frame, thereby interfering with the apparent motion signal of the dots. We used this stimulus with limited lifetime of the points, since we wanted to avoid potential confounds with adaptation at the level of local motion signals. Since the points of a classical pointlight walker move along well-defined local trajectories, they are likely to induce adaptation of motion detectors along their trajectories. Such adaptation of local motion detectors may lead to adaptation effects for the entire walker, which are, however, not specific to biological motion processing. Since we wanted to look specifically for adaptation to biological body motion and body form, we began with a stimulus that contained no veridical local motion trajectories but only biological body motion. A later experiment (Experiment 3) checked whether similar results could be obtained with a walker containing local motion signalsthat is, one in which the light points were located on the major joints of the body-following Johansson (1973).

In a further experiment (Experiment 5), we isolated the effect of local motion signals in a stimulus that contained local motion but no human body form. For this experiment, the starting positions of the light points were randomized, while the motion trajectory of each light point remained intact. Thus, this position-scrambled stimulus contained the veridical local motion trajectories of a human walker, but the body form was destroyed.

The stimuli were presented in a dimly lit room on an Iiyama monitor with a resolution of $1,024 \times 768$ pixels and a display size of $40 \times 30 \mathrm{~cm}$. The monitor refresh rate was $120 \mathrm{~Hz}$. In Experiments 1 and 6, both adaptation and test stimuli were presented in the middle of the screen. The distance of the subjects to the monitor was approximately $70 \mathrm{~cm}$. Stimulus height was $7^{\circ}$. In all the other experiments, adaptation and test stimuli were presented in different screen locations, one in the upper half of the screen and the other in the lower half of the screen. The stimuli in those experiments had to be smaller because two stimuli had to be fitted on the screen. Stimulus height was $3^{\circ}$. The point 
lights were antialiased, 6 pixels in diameter, and the brightness of the points was $85 \mathrm{~cd} / \mathrm{m}^{2}$.

Adaptation stimulus The adaptation stimulus was a forward-walking, rightward- or leftward-facing walker in the facing-adaptation condition (Fig. 2a) or a rightward-facing, forward- or backward-walking walker in the walking-adaptation condition (Fig. 2b). In all the experiments but the third, the number of points on the body of the walker was 4 in each frame of the animation. Their location on the limbs was randomized for each frame. The frame duration was $8.3 \mathrm{~ms}$. This combination of parameters gives a stable impression of a walking figure (Beintema et al., 2006), and all subjects were able to recognize it easily. In Experiment 3, a point-light walker with 12 points on the major joints was used.

Test stimulus The test stimuli were chimeric ambiguous walkers (Thornton, 2003). They consisted of two superimposed walkers with opponent facing directions in the facing-adaptation condition (Fig. 2c) or opponent walking directions in the walking-adaptation condition (Fig. 2d). The light points were distributed over the limbs of the two superimposed walkers. In all but the third experiment, 20 light points were displayed in each animation frame. a

facing direction:

rightward
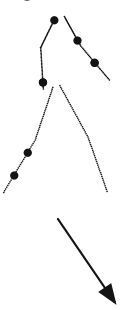

C
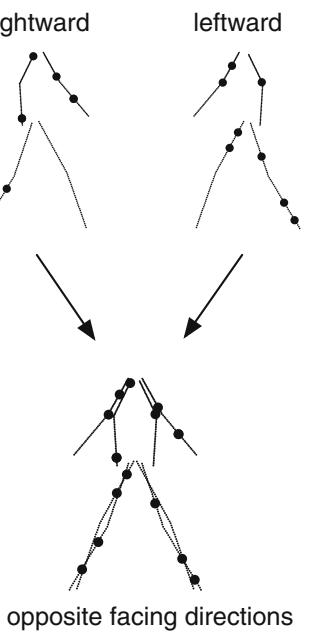

b

d
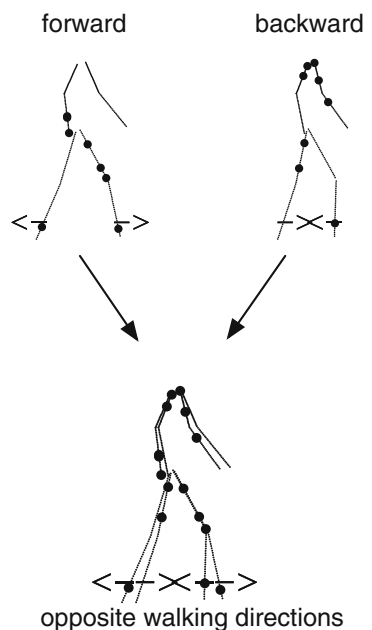

Fig. 2 Adaptation stimuli (a and $\mathbf{b}$ ) and test stimuli (c and $\mathbf{d})$. a In the facing-adaptation condition, the stimulus was either a rightward-facing or a leftward-facing walker. b In the walking-adaptation condition, the adaptation stimulus was either a forward-walking or a backwardwalking walker. $\mathbf{c}$ The test stimulus in the facing-adaptation condition consisted of two superimposed walkers with opposite facing directions, one facing to the right and the other facing to the left. $\mathbf{d}$ In the walking-adaptation condition, the test stimulus consisted of two superimposed walkers with opposite walking directions, one walking forward and the other walking backward. Lines are for illustration purposes only. In the experiments, only the points were shown
Frame duration was $8.3 \mathrm{~ms}$. The distribution of the light points over the limbs of the two walkers was randomized for each frame. By varying the ratio of the distribution of the points between the two superimposed walkers, we varied the degree of ambiguity of the chimera. For example, when more points were located on the body of the rightward-facing walker than on the body of the leftward-facing walker, the rightward-facing walker was perceived more strongly, and subjects were more likely to report rightward facing as the facing direction of the stimulus. The percentage of points located on the limbs of the rightward walker thus describes the degree of ambiguity of the stimulus and will be used to quantify the strength of the aftereffect. Likewise, the percentage of points located on the limbs of the forward-walking walker will be used to assess the degree of ambiguity of the test stimulus for the walking-adaptation condition.

In Experiment 3, a similar procedure was used with point-light walkers with light points on the major joints of the body. In this experiment, the location of the points did not change from one frame to the other but was fixed for the whole stimulus duration. A total of 12 light points were distributed between both walkers of the test stimulus. Thus, the whole walker, which consisted of 12 points, was not always shown. For example, when the percentage of rightward points was $33.3 \%$, the rightward-facing walker had 4 points, while the leftward-facing walker had 8 points. The 4 points of the rightward-facing walker were located on some of the joints of the body for the whole presentation duration.

\section{Procedure}

Subjects always started with the facing-adaptation condition and then participated in the walking-adaptation condition. On each trial, the adaptation stimulus was shown for $20 \mathrm{~s}$. Immediately thereafter, the test stimulus appeared. Subjects had to report their first impression of the facing or walking direction of the test stimulus. The test stimulus was presented until the subject pressed the response button. If the subject did not respond after $1 \mathrm{~s}$, the test stimulus disappeared. Subjects were requested to keep fixation on a central fixation cross throughout the stimulus presentation.

Experiments 1, 2, and 4 contained 20 repetitions for each point ratio and adaptation direction. The total number of 440 trials was grouped in blocks of 22 trials containing each condition once. Experiment 3 contained 32 repetitions for each point ratio and adaptation direction. The total number of 448 trials was grouped in blocks of 28 trials containing each condition twice. Experiment 5 contained 10 repetitions for each point ratio and adaptation direction. The total number of 220 trials was grouped in blocks of 44 trials 
containing each condition twice. In Experiment 6, each point ratio and adaptation direction were repeated 20 times. The total number of 440 trials was grouped in blocks of 44 trials containing each condition twice.

Facing adaptation In the facing-adaptation condition, the adaptation stimulus was facing to the right or facing to the left. On each trial, a rightward-facing walker or a leftwardfacing walker was presented. The facing direction was chosen pseudorandomly. The test stimulus contained two superimposed walkers with different facing directions. The percentage of the rightward points was pseudorandomly chosen out of 11 (or 7 in Experiment 3) different, equidistant values between 0 and 100. Subjects had to report their first impression of the facing direction of the test stimulus by pressing the left arrow button or the right arrow button, respectively.

Walking adaptation In the walking-adaptation condition, the adaptation stimulus was walking forward or backward. On each trial, either a forward- or a backwardwalking walker was chosen pseudorandomly. The test stimulus contained two superimposed walkers with different walking directions. The percentage of the forward points was pseudorandomly chosen out of 11 (or 7 in Experiment 3) different, equidistant values between 0 and 100. Subjects had to report their first impression of the walking direction of the test stimulus by pressing the up arrow button when they wanted to answer forward walking or the down arrow button when they wanted to answer backward walking.

\section{Data analysis}

By estimating the response percentage $p$ against the point percentage, we obtained the psychometric function and determined the point of subjective equality (PSE) for each adaptation direction. We expected that adaptation in one direction would shift the psychometric curve for the test stimulus in the opposite direction. To determine the strength of the aftereffect, we therefore measured the difference between the PSEs for the two adaptation directions (rightward/leftward in the facing-adaptation condition and forward/backward in the walking-adaptation condition). For each subject and adaptation direction, the response data were fitted with a sigmoid logistic function,

$p(x)=\left(1+\mathrm{e}^{\left(-\frac{x-a}{b}\right)}\right)^{-1}$,

and the parameters $a$ and $b$ were estimated to compute the PSEs for the two adaptation directions. Since we expected that adaptation in one direction would shift the PSE in the opposite direction, we used a one-tailed nonparametric
Wilcoxon test for paired samples for the statistical analysis. The strength of the aftereffect was calculated as the differences between the PSEs. In the facing-adaptation task, we expected the difference between rightward and leftward adaptation to be positive. In the walkingadaptation task, we expected the difference between forward and backward adaptation to be positive.

\section{Baseline measurements}

Since the data in the two adaptation conditions turned out to sometimes show biases of both adaptation directions away from a PSE of $50 \%$, we subsequently collected baseline data in sessions without adaptation. In these measurements, subjects saw the chimeric test stimuli without prior adaptation stimuli and had to decide the facing or walking direction of the stimulus. The features of the test stimuli were the same as those in the corresponding adaptation experiment. The test stimulus was presented until the subject pressed the response button. If the subject did not respond after $1 \mathrm{~s}$, the test stimulus disappeared. Immediately after the response was given, the next stimulus appeared. Every subject participated in the baseline measurement task immediately after he or she completed the whole adaptation task. The baseline measurements were recorded for every experiment.

\section{Subjects}

Six subjects, 5 of them female (mean age $=28$ years, $S D=2$ ), participated in Experiment 1. Ten subjects, 7 of them female (mean age $=32$ years, $S D=11$ ), participated in Experiments 2 and 4. In Experiment 3, 10 new subjects, 9 of them female (mean age $=22$ years, $S D=3$ ), participated. In Experiment 5,5 females and 1 male (mean age $=22$ years, $S D=4$ ) participated in the facing-adaptation condition, and 4 females and 2 males (mean age $=21$ years, $S D=3$ ) participated in the walking-adaptation condition. Four females and 2 males (mean age $=25$ years, $S D=4$ ) participated in Experiment 6 . All subjects had normal or corrected-to-normal vision and reported recognizing a walking human when they saw the stimuli. All subjects were naive as to the aims of the experiments, except author S.T., who participated in Experiments 1, 2, 3, 4, and 6.

\section{Results}

Experiment 1: Facing and walking aftereffects

Experiment 1 was intended to establish the facing-adaptation aftereffect and the walking-adaptation aftereffect. Subjects viewed an adaptation stimulus and were then shown a test stimulus that was ambiguous in the facing direction 
or the walking direction. Both adaptation and test stimuli were shown at the same central position on the screen. Facing and walking direction conditions were run in different blocks.

Figure 3a shows the psychometric functions for the facing-adaptation task for the two adaptation directions. The psychometric functions were obtained by plotting the percentages of the answer "rightward" $p(\mathrm{R})$ against the percentage of the rightward-facing points for the collapsed data over all subjects. There was a clear aftereffect, since the psychometric function for rightward
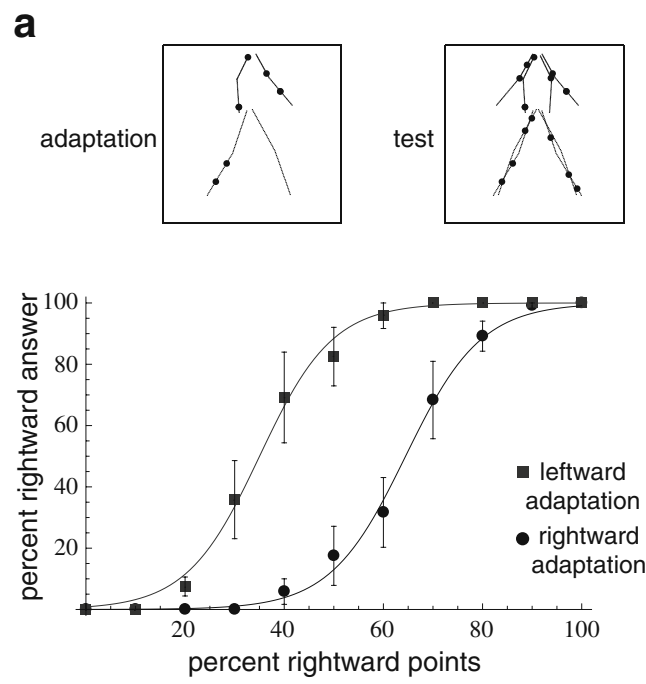

b
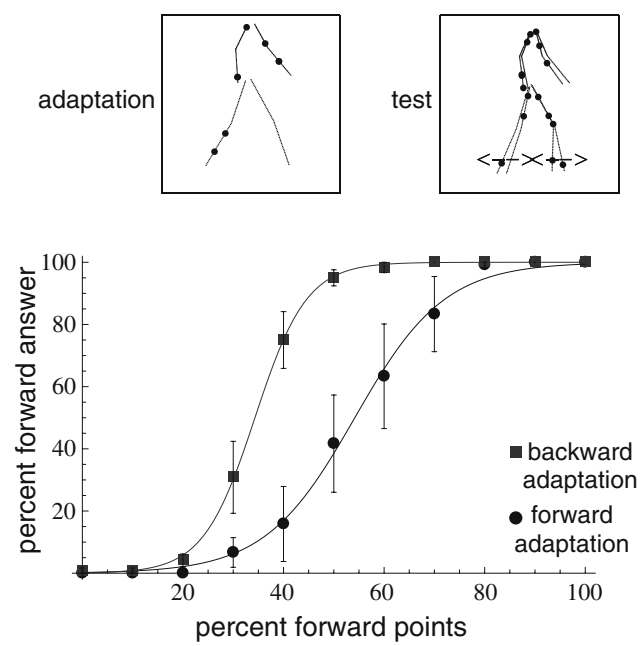

Fig. 3 Results for Experiment 1: Facing adaptation and walking adaptation. a Facing-adaptation task. The psychometric function shows the percentage of rightward-facing choices as a function of the percentage of the points belonging to the rightward segments of the chimeric test stimulus. b Walking-adaptation task. The psychometric function shows the percentage of forward-walking choices as a function of the percentage of the points belonging to the forward segments of the chimeric test stimulus. Error bars represent the standard errors adaptation was shifted to a higher percentage of the rightward-facing points, as compared with the psychometric function for leftward adaptation. The mean difference of the PSEs for leftward- and rightwardfacing adaptors, calculated for every subject individually, was 28 . The PSE value after rightward adaptation $(M=64)$ was significantly higher than the PSE value after leftward adaptation $(M=36), Z=-2.201, p=.01$.

The walking-adaptation task also showed a clear aftereffect (Fig. 3b). The psychometric function for the walking-adaptation task was obtained by plotting the percentage of the answer "forward" $p(\mathrm{~F})$ against the percentage of the forward-walking points for the collapsed data over all subjects. The psychometric function for forward adaptation was shifted to a higher percentage of the forward-walking points, as compared with the psychometric function for backward adaptation. The mean difference of the PSEs for backward- and forward-walking adaptors, calculated for every subject individually, was 20. The PSE value after forward adaptation $(M=55)$ was significantly higher than the PSE value after backward adaptation $(\mathrm{M}=35), Z=-1.992, p=.02$.

Figure 3 thus shows a clear dependency of the direction judgments of the chimeric stimuli on the previous facing and walking directions. The psychometric functions, however, did not appear to lie symmetrically around 50\%. This could indicate a bias for the perception of the chimeric walker or a difference in the strength of the aftereffects of the different facing and walking directions. To determine whether our subjects exhibited a bias in the perception of the chimeric walker even without adaptation, we measured the PSEs for facing and walking discrimination of the chimeric walker in a control experiment in which the chimeric walker was presented without prior adaptation. The result of this control experiment showed that the perception of the chimeric walker was unbiased. For the facing discrimination, the average PSE was 49. For the walking direction discrimination, the average PSE was 51. The asymmetric shift of the psychometric functions in the adaptation experiments therefore suggests a difference in strength of the aftereffects between left and right facing and between forward and backward walking.

Finally, Fig. 3 indicated a difference in strength between the aftereffect for facing and that for walking direction. For facing direction, the difference between the PSEs for the two adaptation directions was 28. For walking direction, the difference was 20. Thus, the strength of the aftereffect was greater in the facingadaptation task than in the walking-adaptation task.

Experiment 2: Different retinal locations of adaptor and test

It is unlikely that the aftereffects observed in Experiment 1 resulted from adaptation of local motion detectors, because 
the stimuli consisted of point-light walkers with limited point lifetime, which limits veridical local image motion signals (Beintema et al., 2006; Beintema \& Lappe, 2002). Spurious and nonveridical local motion signals introduced by this technique (Beintema et al., 2006; Casile $\&$ Giese, 2005) vary randomly from frame to frame and should not lead to any local motion adaptation. However, a second way to avoid adaptation to local motion influencing the results is to present the adaptation and the test stimuli at different retinal locations, since local motion adaptation at one position in the visual field is known to transfer only weakly to other positions (Mather et al., 2008; Moulden, 1980).

When adaptation and test stimuli were presented at different locations, there was still an aftereffect for facing adaptation (Fig. 4a). Again, the psychometric function for rightward adaptation was shifted to a higher percentage of the rightward-facing points, as compared with the psychometric function for leftward adaptation. The mean difference of the PSEs for leftward- and rightward-facing adaptors, calculated for every subject individually, was 11 . The PSE value after rightward adaptation $(M=55)$ was significantly higher than the PSE value after leftward adaptation $(M=44), Z=-2.703, p=.004$.

There was also an aftereffect in the walking-adaptation task. The psychometric function for forward adaptation was shifted, as compared with the psychometric function for backward adaptation (Fig. 4b). The mean difference of the PSEs for backward- and forward-walking adaptors, calculated for every subject individually, was 6 . The PSE value after forward adaptation $(M=45)$ was significantly higher than the PSE value after backward adaptation $(M=$ 39), $Z=-1.661, p=.049$. As in Experiment 1, the perception of the chimeric walker in the control condition was unbiased. The PSE was 52 for facing discrimination and 47 for walking direction discrimination. Also as in Experiment 1, the strength of the aftereffect was greater in the facingadaptation task than in the walking-adaptation task.

We conclude that the facing-adaptation aftereffect and the walking-adaptation aftereffect appear even when adaptation and test stimuli are presented at different retinal locations.

The strengths of the aftereffects - that is, the differences between the PSEs in the two adaptation directions-were smaller than in Experiment 1. This decrease in strength might be related to the change in position between adaptor and test and might thus show that the aftereffects are only partly position invariant. Similar decreases in adaptation strength with difference in position of adaptation and test stimuli have also been observed in other complex pattern aftereffects (e.g., Meng, Mazzoni, \& Qian, 2006). Alternatively, the strength of the aftereffects might have been smaller than in Experiment 1 because the stimuli were
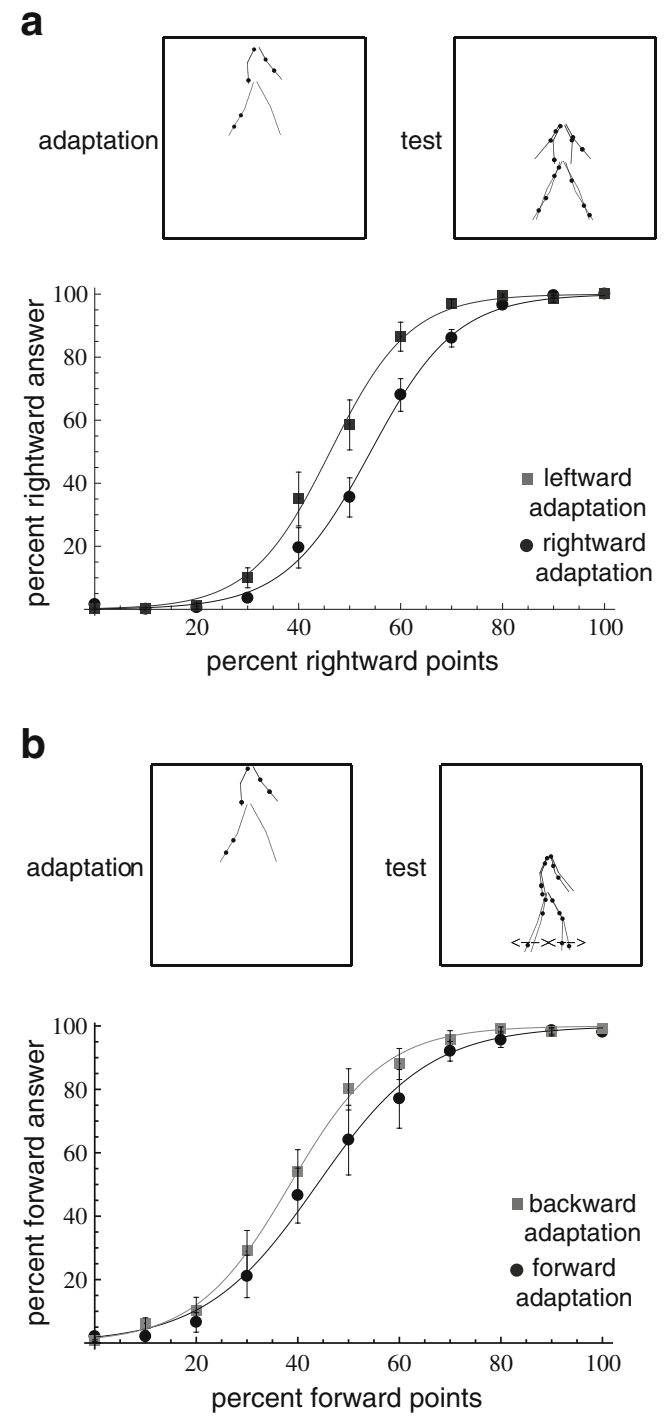

Fig. 4 Results for Experiment 2: Facing adaptation and walking adaptation for stimuli presented in different retinal locations. a Facingadaptation task. The psychometric function shows the percentage of rightward-facing choices as a function of the percentage of the points belonging to the rightward segments of the chimeric test stimulus. b Walking-adaptation task. The psychometric function shows the percentage of forward-walking choices as a function of the percentage of the points belonging to the forward segments of the chimeric test stimulus. Error bars represent the standard errors

smaller. In Experiment 1, the stimuli were more than twice as big as the stimuli used in this experiment.

\section{Experiment 3: Classic point-light walker}

In Experiment 3 we wanted to confirm the findings of a facing-adaptation and a walking-adaptation aftereffect for the classic point-light walker of Johansson (1973). Since the results in the experiment above had shown that both aftereffects can be observed when adaptor and test were administered at different retinal positions, we used different 
locations for adaptation and test stimuli to avoid adaptation effects arising simply from local adaptation of local image motion of the point-lights.

The adaptation stimulus was a classic point-light walker with 12 light points on the major joints of the body. The chimeric test stimulus consisted of two superimposed walkers with a total number of 12 points located on the joints. In the most extreme cases of the test stimulus point distributions, all 12 points of the test stimulus were located on just one of the two walkers. In the intermediate cases, a certain number of points were located on one walker, and the remainder on the other walker - in each case, on some randomly selected joints.

In the results of the facing-adaptation task, the psychometric function for rightward adaptation was shifted to a higher percentage of the rightward-facing points, as compared with the psychometric function for leftward adaptation (Fig. 5a). The mean difference of the PSEs for leftwardand rightward-facing adaptors, calculated for every subject individually, was 15 . The PSE value after rightward adaptation $(M=58)$ was significantly higher than the PSE value after leftward adaptation $(M=43), Z=-2.807, p=$ .003. The PSE for the chimera walker without prior adaptation was 55 .

There was also a shift between the psychometric function for the forward-adaptation task, as compared with the psychometric function for the backward-adaptation task (Fig. 5b). The mean difference of the PSEs for backward- and forward-walking adaptors, calculated for every subject individually, was 7 . The PSE value after forward adaptation $(M=44)$ was significantly higher than the PSE value after backward adaptation $(M=37), Z=-2,016, p=$ .02. The PSE for the chimera walker without prior adaptation was 46.

We conclude that the facing-adaptation aftereffect and the walking-adaptation aftereffect can also be observed when classic point-light walkers are used. The strength of the aftereffects was similar to those obtained with the limited-lifetime stimuli.

Experiment 4: Facing adaptation with randomized walking sequences

In the two-stage model shown in Fig. 1, the facing and the walking aftereffects are derived from different neuronal representations, a first one representing body form and a second one representing body motion. This predicts that the body form aftereffect should not rely on the presence of body motion. Indeed, the detection of the facing direction is possible even from static pictures of a pointlight walker (Reid, Brooks, Blair, \& van der Zwan, 2009) or when the frames of the stimulus are temporally scrambled and, thus, the stimulus does not contain a valid
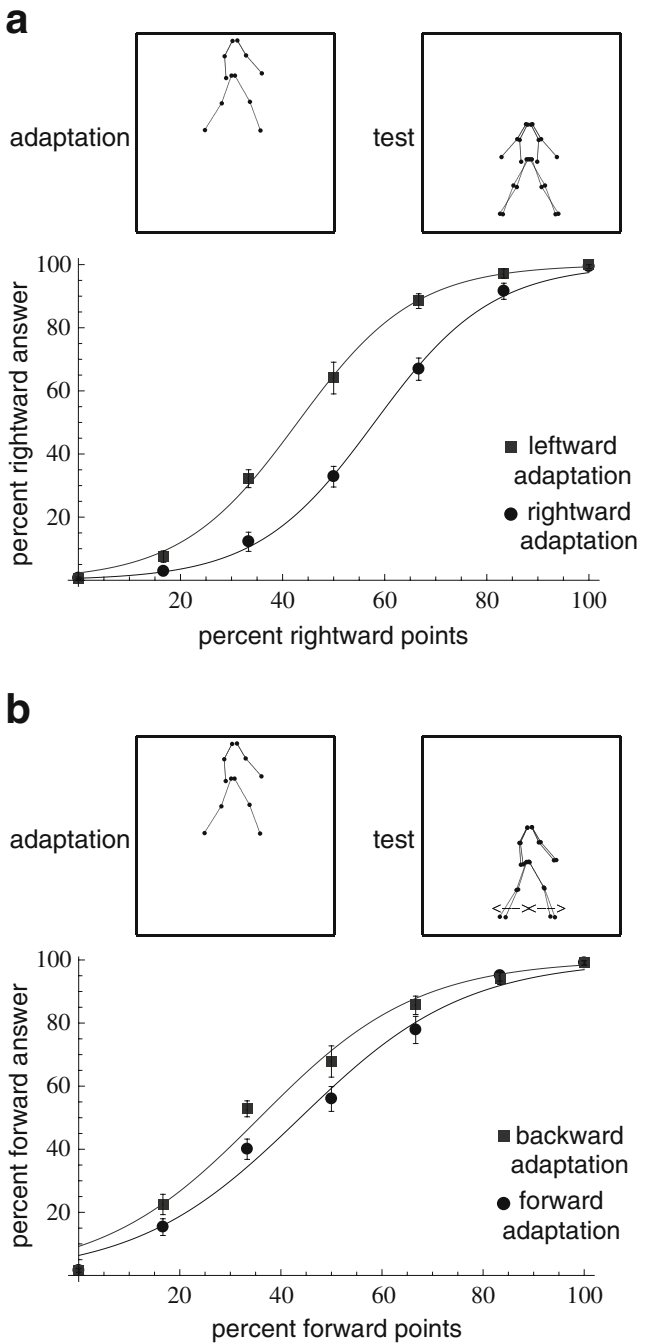

Fig. 5 Results for Experiment 3: Facing adaptation and walking adaptation for classic point-light walkers presented in different retinal locations. a Facing-adaptation task. The psychometric function shows the percentage of rightward-facing choices as a function of the percentage of the points belonging to the rightward segments of the chimeric test stimulus. b Walking-adaptation task. The psychometric function shows the percentage of forward-walking choices as a function of the percentage of the points belonging to the forward segments of the chimeric test stimulus. Error bars represent the standard errors

motion sequence (Lange \& Lappe, 2007). We thus reasoned that if the facing-adaptation aftereffect results from the adaptation to the form of the stimulus, coherent walking motion should not be necessary to show a facingadaptation aftereffect. We tested this by measuring the facing-adaptation aftereffect when both adaptation and test stimuli were temporally scrambled walker sequencesthat is, when the normal temporal order of the frames was randomized. In these stimuli, for each frame of the animation, a random phase of the walking cycle was chosen. Thus, each frame displayed a single body posture, but the sequence in which these body postures were 
shown was random and, thus, did not induce a body motion perception.

We observed an aftereffect for facing adaptation in the absence of natural body motion. The psychometric function for rightward adaptation was shifted, as compared with the psychometric function for leftward adaptation (Fig. 6). The mean difference of the PSEs for leftward- and rightwardfacing adaptors, calculated for every subject individually, was 8 . The PSE value after rightward adaptation $(M=54)$ was significantly higher than the PSE value after leftward adaptation $(M=46), Z=-2.448, p=.007$. The PSE without prior adaptation was 51 .

We conclude that facing adaptation occurred even when the stimulus sequence contained body form information in random order. The strength of the aftereffect was comparable to that observed with a normal movement sequence in Experiment 2. Thus, the natural motion of the limbs is not necessary to show aftereffects in biological motion adaptation. The adaptation therefore must be due to adaptation of template cells at the body form analysis level and is independent of the body motion analysis level.

\section{Experiment 5: Position scrambled adaptation stimulus}

The experiment above showed that body form information is sufficient to generate a facing direction aftereffect in biological motion. This was shown by removing the body motion sequence information from the adaptor while retaining the body form information. Experiment 5 used a similar approach to test whether local motion trajectories are sufficient to induce biological motion aftereffects. This

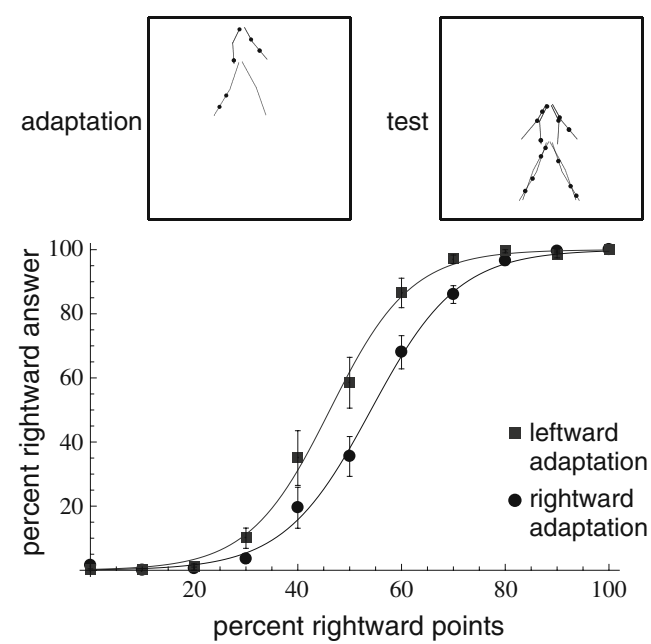

Fig. 6 Results for Experiment 4: Facing adaptation for stimuli with random temporal order. The psychometric function shows the percentage of rightward-facing choices as a function of the percentage of the points belonging to the rightward segments of the chimeric test stimulus. Error bars represent the standard errors was done by removing the body form information from the adaptor while retaining the local motion trajectories. We used an adaptation stimulus in which each dot followed the veridical motion trajectory of a joint of the classic Johansson walker (Johansson, 1973), but starting from a random position within the area of the screen that was covered by the walker adaptor stimuli in Experiments 2-4. This stimulus contains the typical motion trajectories of a walker but no body form. If body form analysis is necessary for the facing direction aftereffect, we would expect that the facing aftereffect would vanish. Moreover, if body form analysis precedes body motion analysis and is necessary for the global body motion analysis, we also would expect the walking direction aftereffect to vanish.

Figure 7 shows the results of the facing and the walking direction discrimination after the respective adaptations. The mean difference of the PSEs for leftward- and rightward-facing adaptors, calculated for every subject individually, was -0.2 . The PSE value after rightward adaptation $(M=50.8)$ was not significantly higher than the PSE value after leftward adaptation $(M=51), Z=$ $0.000, p>.9$.

The mean difference of the PSEs for backward- and forward-walking adaptors, calculated for every subject individually, was -4 . The PSE value after forward adaptation $(M=46)$ was not significantly higher than the PSE value after backward adaptation $(M=50), Z=-0.841, p=.2$.

We conclude that position-scrambled point-light stimuli, which contain veridical local motion trajectories of a human walker but no body form, are not sufficient to induce a facing- or a walking-adaptation aftereffect. Thus, body form is necessary for the facing- and the walking-adaptation aftereffects.

Experiment 6: Facing adaptation and walking direction discrimination

In the previous experiments we observed a body form aftereffect based on the adaptation of the body form analysis stage and a body motion aftereffect based on the adaptation of the body motion analysis stage. In Experiment 6, we investigated the interaction between the two stages. We asked whether adaptation at the body form stage can influence decisions on the body motion stage, as hierarchical processing would predict.

We tested the influence of body form adaptation on the walking direction decision. The adaptation stimulus was a time-scrambled walker facing to the right or to the left, similar to the adaptor in Experiment 4, which did not contain veridical body motion but only veridical body form. The stimulus adapts templates at the body form stage, as shown in Experiment 4, but should not lead to adaptation at the body motion stage, because it contains no body motion. 

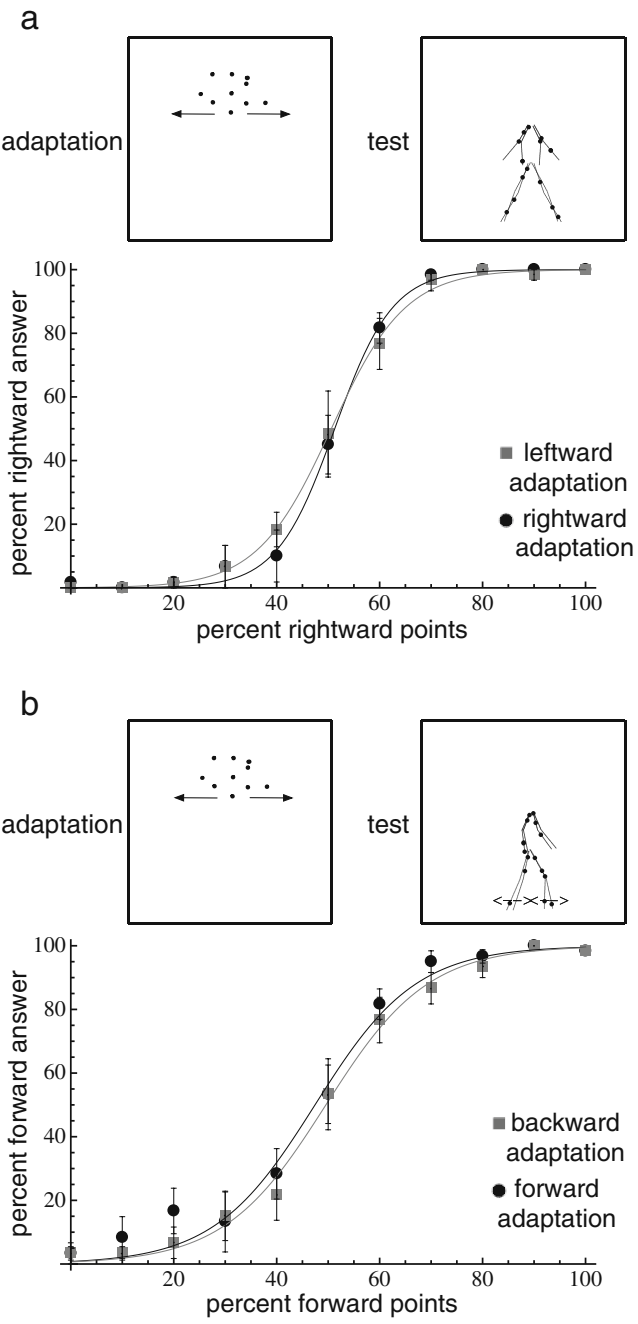

Fig. 7 Results for Experiment 5: Facing adaptation and walking adaptation for position-scrambled adaptor stimuli. a Facing-adaptation task. The psychometric function shows the percentage of rightwardfacing choices as a function of the percentage of the points belonging to the rightward segments of the chimeric test stimulus. b Walkingadaptation task. The psychometric function shows the percentage of forward-walking choices as a function of the percentage of the points belonging to the forward segments of the chimeric test stimulus. Error bars represent the standard errors

We used the adaptation at the body form stage to test whether the discrimination of walking direction is based on sequence analysis of body postures, as depicted in Fig. 1. If the walking direction computation is based on template activities in the body form stage, adaptation of the body form stage for a particular facing direction should affect the perception of walking direction for that facing direction, but not for the opposite facing direction.

To test this prediction, the test stimulus was composed of two superimposed walkers, one facing to the left and one facing to the right. One of these walkers was walking forward, the other was walking backward. Subjects had to report the walking direction of the test stimulus.
Figure 8 shows the results. Two psychometric curves show the percentages of forward answers as a function of the percentage of forward points in the chimeric test stimulus. One of the curves gives the data (shown by circles) for the conditions in which the adaptation stimulus and the forward-walking walker of the chimeric test stimulus faced in the same direction. The other (squares) shows the conditions in which the adaptation stimulus and the forward-walking walker of the chimeric test stimulus faced in opposite directions. The difference between the two curves shows the influence of the facing-adaptation on the decision in the walking discrimination task. When the adaptation stimulus and the forward-walking walker of the chimeric test stimulus faced in the same direction, the PSE for forward walking was shifted to higher percentages of forward points than when the adaptation stimulus and the forward-walking walker of the test stimulus faced in the opposite direction. The mean difference of the PSEs for same and opposite facing directions, calculated for every subject individually, was 4 . The PSE value for same facing directions $(M=51)$ was significantly higher than the PSE value for opposite facing directions $(M=46), Z=-2.032, p=.02$. The PSE without prior adaptation was 48.

We conclude that facing adaptation at the body form stage shifts the balance for the walking direction decision such that same-facing walkers need more signal points to be perceived in equal strength. Since the time-scrambled adaptor stimulus should not lead to adaptation at the body

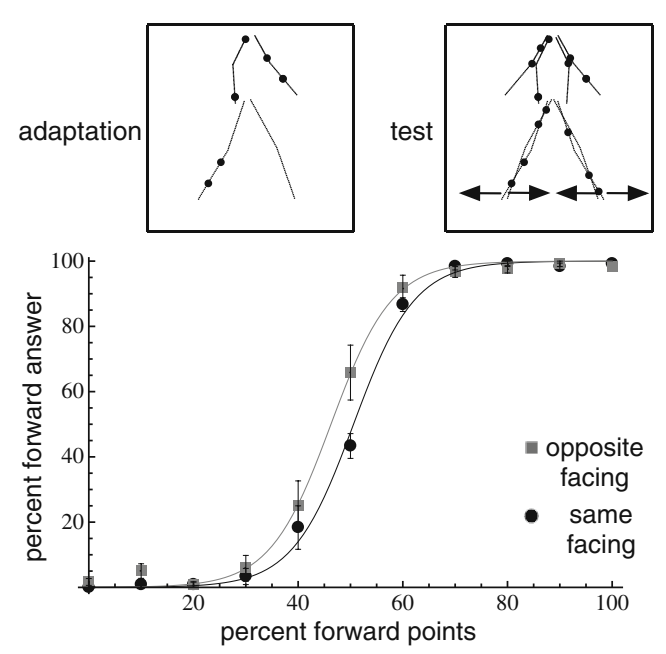

Fig. 8 Results for Experiment 6: Effect of the facing adaptation on the walking direction decision. The psychometric function shows the percentage of forward-walking choices as a function of the percentage of the points belonging to the forward segments of the chimeric test stimulus. Subjects adapted to the facing direction. The adaptor and the forward-walking walker of the chimeric test stimulus could face in the same direction (circles) or in opposite directions (squares). Error bars represent the standard errors 
motion analysis stage, we further conclude that the analysis of walking direction is based on the template activities at the body form stage.

\section{Discussion}

We have studied two novel aftereffects for biological motion. The first, adaptation to the facing direction of a stimulus, leads to a more intensive perception of the opponent facing direction of an ambiguous test stimulus. The second, adaptation to the walking direction of a stimulus, leads to a more intensive perception of the opponent walking direction of an ambiguous test stimulus.

We suggest that the facing-adaptation aftereffect and the walking-adaptation aftereffect are two separate effects based on two separate processing levels of biological motion (Beintema \& Lappe, 2002; Giese \& Poggio, 2003; Lange \& Lappe, 2006). The facing-adaptation aftereffect is based on the body form analysis level, a processing level that uses only the form and not the motion of the walker. This body form analysis might be performed by form template cells. Such template cells can likely be found in fusiform (Michels, Lappe, \& Vaina, 2005; Peelen \& Downing, 2005b) or occipital (Grossman \& Blake, 2002; Michels et al., 2005; Peelen \& Downing, 2005a; Vaina, Solomon, Chowdhury, Sinha, \& Belliveau, 2001) shape-selective areas, the extrastriate body area (Downing, Jiang, Shuman, \& Kanwisher, 2001), or parts of the superior temporal sulcus (Vangeneugden et al., 2011; Vangeneugden, Pollick, \& Vogels, 2008). The walkingadaptation aftereffect, in contrast, must be based on body motion analysis, a processing level that derives information from the body form analysis level and, thus, uses both form and motion of the stimulus. The body motion is analyzed by neurons that evaluate the temporal order of signals from the body form analysis level. They are likely to be found in the superior temporal sulcus (STS; Beauchamp, Lee, Haxby, \& Martin, 2002; Bonda, Petrides, Ostry, \& Evans, 1996; Grossman et al., 2000; Oram \& Perret, 1994, 1996; Puce \& Perrett, 2003; Santi, Servos, Vatikiotis-Bateson, Kuratate, \& Munhall, 2003; Thompson, Clarke, Stewart, \& Puce, 2005; Vaina et al., 2001; Vangeneugden et al., 2011; Vangeneugden et al., 2008).

Although our experiments were guided by the body form analysis process proposed by Beintema and Lappe (2002) and Lange and Lappe (2006), as well as in the body form pathway of Giese and Poggio (2003), we have to consider any possible role for local motion analysis. Formal models of local motion contributions to biological motion perception were proposed early on in the form of vector-coding models (Cutting, 1981; Johansson, 1973, 1976). These models rely on the relative motion of the joints with respect to each other and, thus, on the presence of point-lights on all major joints. The same is true for models that use local constraints in the limb movement, particularly through the restrictions of the knees and elbows (Hoffman \& Flinchbaugh, 1982; Webb \& Aggarwal, 1982). Studies in which the point-lights were placed on the limbs between the joints (Beintema \& Lappe, 2002; Cutting, 1981; Dittrich, 1993) or in which some joints were omitted (Mather, Radford, \& West, 1992; Neri, Morrone, \& Burr, 1998; Pinto \& Shiffrar, 1999) have shown that point-lights need not be placed on the joints for biological motion perception.

The model of Giese and Poggio (2003) includes, in addition to a body form pathway for full-body motion, a motion pathway that determines the facing and the walking direction of point-light stimuli by three consecutive motion processes: local motion, opponent motion, and optic flow patterns akin to motion template cells. The opponent motion pathway of that model also works to some degree for limited-lifetime stimuli (Casile \& Giese, 2005), although only for the facing task and without capturing the dependence on lifetime and number of dots found in human observers (Beintema \& Lappe, 2002). If, however, the aftereffects we observed were related to adaptation of opponent motion mechanisms, one would expect them to be stronger for classic point-light walkers than for limitedlifetime walkers and of equal strength for facing and walking adaptation, because both would be based on the same opponent motion mechanism. In our results, the aftereffects for classic and limited-lifetime walkers were of the same strength, and the aftereffects for facing were typically stronger than those for walking direction. This is consistent with a model in which the walking direction is derived from body form templates.

The facing-adaptation aftereffect and the walkingadaptation aftereffect were observed when adaptor and test were in different retinal locations. This is similar to the position invariance found in the face aftereffect (Leopold et al., 2001; Watson \& Clifford, 2003) or in adaptation effects to other biological motion stimuli (Jackson \& Blake, 2010). The position-invariant face aftereffect primarily involves the fusiform face area (Kovacs et al., 2008), a cortical area that is also active in the perception of biological motion (Michels et al., 2005; Peelen \& Downing, 2005b) and that may be responsible for the body form matching stage (Giese \& Poggio, 2003; Lange \& Lappe, 2006).

Position-invariant motion aftereffects have been observed in complex motion stimuli (Hershenson, 1984; Meng et al., 2006; Price, Greenwood, \& Ibbotson, 2004; Snowden \& Milne, 1997) that activate body motion processing areas such as the middle temporal area and the medial superior temporal area. In contrast, local motion aftereffects, such as the adaptation to first-order motion, are not position invariant 
(Mather et al., 2008; Moulden, 1980). To avoid adaptation to the local motion of the stimulus, we used walkers in which the positions of the points were distributed over the whole body and changed from one frame to the next. When presenting the adaptation and the test stimuli at different locations, we also used the classic point-light walker with points positioned on the joints of the body.

If local motion adaptation enhances facing and walking adaptation, one would expect a greater effect for the classic point-light walker (Experiment 3), in comparison with a walker in which the position of the points on the body change from one frame to the next (Experiment 2). The strengths of the facing-adaptation effect and the walkingadaptation effect did not differ between these experiments. Thus, local image motion signals did not enhance the aftereffects. Furthermore a position-scrambled adaptor that contained the same point-light motions as a walker, but not a human form, evoked neither a facing nor a walking aftereffect, even though it is known that humans are capable of discriminating the walking direction of a spatial scrambled stimulus in a two-alternative forced choice task by focusing on the trajectories of the feet (Troje \& Westhoff, 2006).

Instead, our experiments provide further information about the ways in which body form information is used in biological motion perception. Randomizing the temporal order of the frames of the stimulus removes body motion information. This temporally scrambled stimulus contains only form information about body postures, and only the facing direction, but not the walking direction, can be determined (Hirai \& Hiraki, 2006; Lange \& Lappe, 2007). Experiment 4 showed that the facing-adaptation aftereffect also exists when a temporally scrambled adaptation stimulus is used. Thus, adaptation to the facing direction is not dependent on body motion. Adaptation to body form is sufficient to evoke the facing-adaptation aftereffect.

Experiment 6 showed that adaptation to body form also influences body motion analysis. This is an important point since it relates to the hierarchical analysis of form and motion of the human body. Adaptation to the facing direction of a temporally scrambled walker-that is, a stimulus that did not convey body motion-affected the decision in a walking direction discrimination between stimuli facing in the same and stimuli facing in the opposite direction as the adaptor. This shows that the analysis of the body form is subsequently used for the analysis of the body motion-that is, that body form analysis precedes body motion analysis.

In Experiments 1-3, the facing-adaptation aftereffect was always greater than the walking-adaptation aftereffect. The hierarchical relation between body form and body motion demonstrated in Experiment 6 may offer an explanation for this difference in strength of the aftereffects.
Exposure to a regular walker, as in Experiments 1-3, induces adaptation both at the body form and at the body motion stages. Because of the adaptation at the body form stage, the input that the body form stage provides to the body motion stage will be weakened, resulting in a weaker overall adaptation at the body motion stage. However, this alone cannot explain the different strengths of the facing and the walking aftereffects, since the aftereffect in Experiment 6, which tested the pure influence of adaptation at the body form stage on the walking discrimination, was smaller than the difference between facing and walking adaptation in Experiment 1. A different explanation for the difference in strengths between the facing and the walking aftereffects might lie in a quantitative difference in their representations. Recently, Vangeneugden et al. (2011) showed that many more neurons in the monkey STS and IT are selective for facing direction than for walking direction. This difference in population size could also lead to different strengths in aftereffects. Another possibility is a difference in the representation between forward and backward walking. If there were less neurons representing backward walking than representing forward walking in the visual system, the walking aftereffect should be smaller than the facing aftereffect.

Such a possible asymmetry in the representation of forward and backward walking may also explain the biases for forward, as compared with backward, walking in Experiments 1-4. The shift between the psychometric curves was not symmetric with respect to the balanced number of points in the chimeric test stimulus. On the other hand, a response bias was also found for the report of rightward facing in Experiments 2-4. While it may seem plausible that forward walking may be better represented in the visual system than backward walking, because it is more commonly observed in everyday life, such an argument is less plausible for rightward than for leftward facing. To search for a possible explanation for the facing direction asymmetry, we additionally tested 1 subject who had shown clear asymmetries in the first experiment with a repetition of the facing adaptation task with a backwardwalking adaptor. This gave no change in the size or in the asymmetry of the effect. Likewise, a repetition of the walking adaptation effect with a leftward-walking adaptor also gave no change in either size or asymmetry. Moreover, separate testing of the perception of the chimeric walker without prior adaptation did not reveal strong biases. The PSEs in the unadapted control experiments were all close to the unbiased value of $50 \%$. Response biases have been observed also in other studies of biological motion aftereffects (Jackson \& Blake, 2010; Troje, Sadr, Geyer, \& Nakayama, 2006) but remain difficult to explain. We therefore think that our comparison of the PSE for rightward versus leftward adaptation or for forward versus 
backward adaptation is the most reliable measure of the adaptation effect.

Previous studies have shown aftereffects for even more complex properties of biological motion stimuli. Troje et al. (2006) investigated adaptation to the gender of a walking figure. Subjects adapted to the gender of a person by watching a male or a female point-light walker. Thereafter, a morphed point-light walker ambiguous in gender was shown. After adaptation to the gender, subjects perceived the opposite gender. A recent study tested the adaptation to the orientation of static pictures of human bodies (Lawson, Clifford, \& Calder, 2009). That study concluded that the orientation of the body is represented in a multichannel coding - that is, that there are neurons for different body postures. This is consistent with the idea of template neurons for different actions and different views of an action. Jackson and Blake (2010) found an adaptation aftereffect also to the facing direction (frontward facing and backward facing).

In summary, our observation of separate aftereffects for facing and walking adaptation provides further evidence that perception of biological motion contains two levels of analysis: body form analysis at a first level and body motion analysis at a second, consecutive level. Since the adaptation aftereffects for the facing-adaptation task were always greater than the adaptation aftereffects for the walking-adaptation task, and since body form analysis contributes to body motion analysis (Experiment 6), the two effects are likely based on hierarchically different cortical levels, supporting the notion that analysis of body form happens before analysis of body motion.

Acknowledgements We would like to thank the reviewers for their insightful comments and helpful suggestions.

\section{References}

Beauchamp, M. S., Lee, K. E., Haxby, J. V., \& Martin, A. (2002). Parallel visual motion processing streams for manipulable objects and human movements. Neuron, 34, 149-159.

Beintema, J. A., Georg, K., \& Lappe, M. (2006). Perception of biological motion from limited lifetime stimuli. Perception \& Psychophysics, 68, 613-624.

Beintema, J. A., \& Lappe, M. (2002). Perception of biological motion without local image motion. Proceedings of the National Academy of Sciences, 99, 5661-5663.

Bonda, E., Petrides, M., Ostry, D., \& Evans, A. (1996). Specific involvement of human parietal systems and the amygdala in the perception of biological motion. Journal of Neuroscience, 16 , 3737-3744.

Casile, A., \& Giese, M. A. (2005). Critical features for the recognition of biological motion. Journal of Vision, 5, 348-360.

Clifford, C. W. G., Webster, M. A., Stanley, G. B., Stocker, A. A., Kohn, A., Sharpee, T. O., \& Schwartz, O. (2007). Visual adaptation: Neural, psychological and computational aspects. Vision Research, 47, 3125-3131.

Cutting, J. E. (1981). Coding theory adapted to gait perception. Journal of Experimental Psychology: Human Perception \& Performance, 7, 71-87.

Dittrich, W. H. (1993). Action categories and the perception of biological motion. Perception, 22, 15-22.

Downing, P. E., Jiang, Y., Shuman, M., \& Kanwisher, N. (2001). A cortical area selective for visual processing of the human body. Science, 293, 2470-2473.

Giese, M. A., \& Poggio, T. (2003). Neural mechanisms for the recognition of biological movements. Nature Reviews Neuroscience, 4, 179-192.

Grossman, E. D., \& Blake, R. (2002). Brain areas active during visual perception of biological motion. Neuron, 35, 1167-1175.

Grossman, E. D., Donnelly, M., Price, R., Pickens, D., Morgan, V., Neighbor, G., \& Blake, R. (2000). Brain areas involved in perception of biological motion. Journal of Cognitive Neuroscience, $12,711-720$.

Hershenson, M. (1984). Phantom spiral effect: Evidence for global mechanisms in perception. Bulletin of the Psychonomic Society, 22, 535-537.

Hirai, M., \& Hiraki, K. (2006). The relative importance of spatial versus temporal structure in the perception of biological motion: An event-related potential study. Cognition, 99, B15-B28.

Hoffman, D. D., \& Flinchbaugh, B. E. (1982). The interpretation of biological motion. Biological Cybernetics, 42, 195-204.

Jackson, S., \& Blake, R. (2010). Neural integration of information specifying human structure from form, motion and depth. Journal of Neuroscience, 30, 838-848.

Johansson, G. (1973). Visual perception of biological motion and a model for its analysis. Perception \& Psychophysics, 14, 201-211.

Johansson, G. (1976). Spatio-temporal differentiation and integration in visual motion perception. Psychological Research, 38, 379 393.

Kovacs, G., Cziraki, C., Vidnyanszky, Z., Schweinberger, S. R., \& Greenlee, M. W. (2008). Position-specific and position-invariant face aftereffects reflect the adaptation of different cortical areas. NeuroImage, 43, 156-164.

Lange, J., Georg, K., \& Lappe, M. (2006). Visual perception of biological motion by form: A template-matching analysis. Journal of Vision, 6, 836-849.

Lange, J., \& Lappe, M. (2006). A model of biological motion perception from configural form cues. Journal of Neuroscience, 26, 2894-2906.

Lange, J., \& Lappe, M. (2007). The role of spatial and temporal information in biological motion perception. Advances in Cognitive Psychology, 3, 419-429.

Lawson, R. P., Clifford, C. W., \& Calder, A. J. (2009). About turn: The visual representation of human body orientation revealed by adaptation. Psychological Science, 20, 363-371.

Leopold, D. A., O'Toole, A. J., Vetter, T., \& Blanz, V. (2001). Prototype-referenced shape encoding revealed by high-level aftereffects. Nature Neuroscience, 4, 89-94.

Mather, G., Pavan, A., Campana, G., \& Casco, C. (2008). The motion aftereffect reloaded. Trends in the Cognitive Sciences, 12, 481487.

Mather, G., Radford, K., \& West, S. (1992). Low-level visual processing of biological motion. Proceedings of the Royal Society of London B, 249, 149-155.

Meng, X., Mazzoni, P., \& Qian, N. (2006). Cross-fixation transfer of motion aftereffects with expansion motion. Vision Research, 46, 3681-3689.

Michels, L., Lappe, M., \& Vaina, L. M. (2005). Visual areas involved in the perception of human movement from dynamic form analysis. NeuroReport, 16, 1037-1041. 
Moulden, B. (1980). After-effects and the integration of patterns of neural activity within a channel. Philosophical Transactions of the Royal Society of London B, 290, 39-55.

Neri, P., Morrone, M. C., \& Burr, D. C. (1998). Seeing biological motion. Nature, 395, 894-896.

Oram, M. W., \& Perret, D. I. (1994). Responses of anterior superior temporal polysensory area STPa neurons to "biological motion" stimuli. Journal of Cognitive Neuroscience, 6, 99-116.

Oram, M. W., \& Perret, D. I. (1996). Integration of form and motion in the anterior superior temporal polysensory area STPa of the macaque monkey. Journal of Neurophysiology, 76, 109-129.

Peelen, M. V., \& Downing, P. E. (2005a). Is the extrastriate body area involved in motor actions? Nature Neuroscience, 8, 125.

Peelen, M. V., \& Downing, P. E. (2005b). Selectivity for the human body in the fusiform gyrus. Journal of Neurophysiology, 93, 603-608.

Pinto, J., \& Shiffrar, M. (1999). Subconfigurations of the human form in the perception of biological motion displays. Acta Psychologica, 102, 293-318.

Price, N. S. C., Greenwood, J. A., \& Ibbotson, M. R. (2004). Tuning properties of radial phantom motion aftereffects. Vision Research, 44, 1971-1979.

Puce, A., \& Perrett, D. (2003). Electrophysiology and brain imaging of biological motion. Philosophical Transactions of the Royal Society of London B, 358, 435-445.

Reid, R., Brooks, A., Blair, D., \& van der Zwan, R. (2009). Snap! Recognising implicit actions in static point-light displays. Perception, 38, 613-616.

Santi, A., Servos, P., Vatikiotis-Bateson, E., Kuratate, T., \& Munhall, K. (2003). Perceiving biological motion: Dissociating visible speech from walking. Journal of Cognitive Neuroscience, 15, 800-809.
Snowden, R. J., \& Milne, A. B. (1997). Phantom motion after effectsevidence of detectors for the analysis of optic flow. Current Biology, 7, 717-722.

Thompson, J. C., Clarke, M., Stewart, T., \& Puce, A. (2005). Configural processing of biological motion in human superior temporal sulcus. Journal of Neuroscience, 25, 9059-9066.

Thornton, I. M. (2003). Last but not least: A chimeric point-light walker. Perception, 32, 377-383.

Troje, N. F., \& Westhoff, C. (2006). The inversion effect in biological motion perception: Evidence for a "life detector"? Current Biology, 16, 821-824.

Troje, N. F., Sadr, J., Geyer, H., \& Nakayama, K. (2006). Adaptation aftereffects in the perception of gender from biological motion. Journal of Vision, 6, 850-857.

Vaina, L. M., Solomon, J., Chowdhury, S., Sinha, P., \& Belliveau, J. W. (2001). Functional neuroanatomy of biological motion perception in humans. Proceedings of the National Academy of Sciences, 98, 11656-11661.

Vangeneugden, J., De Maziere, P. A., Van Hulle, M. M., Jaeggli, T., Van Gool, L., \& Vogels, R. (2011). Distinct mechanisms for coding of visual actions in macaque temporal cortex. Journal of Neuroscience, 31, 385-401.

Vangeneugden, J., Pollick, F., \& Vogels, R. (2008). Functional differentiation of macaque visual temporal cortical neurons using a parametric action space. Cerebral Cortex, 19, 593-611.

Watson, T. L., \& Clifford, C. W. G. (2003). Pulling faces: An investigation of the face-distortion aftereffect. Perception, 32, 1109-1116.

Webb, J. A., \& Aggarwal, J. K. (1982). Structure from motion of rigid and jointed objects. Artificial Intelligence, 19, 107-130.

Webster, M. A., Kaping, D., Mizokami, Y., \& Duhamel, P. (2004). Adaptation to natural facial categories. Nature, 428, 557-561. 